\title{
NOT ALL SEMIREGULAR URYSOHN-CLOSED SPACES ARE KATĚTOV-URYSOHN
}

\author{
JACK R. PORTER 1 \\ Abstract. A topological space is said to be Urysohn if every pair \\ of distinct points have disjoint closed neighborhoods. In this note \\ we give an example of a first countable semiregular Urysohn space \\ which is closed in every Urysohn space in which it can be embedded, \\ and on which there exists neither a coarser minimal Urysohn topol- \\ ogy nor a coarser minimal first countable Urysohn topology.
}

For a topological property $P$, a $P$ space is minimal $P$ if there is no strictly coarser $P$ topology and $P$-closed if it is closed in every $P$ space containing it; a space is Katétov- $P$ if there is a coarser minimal $P$ topology. It is known [1], [2], [3], [6], [8], [9], [10] that a $P$-closed space is Katětov- $P$ whenever $P=$ Hausdorff, first countable Hausdorff, completely Hausdorff, first countable regular, completely regular, first countable completely regular, normal, first countable normal, completely normal, first countable completely normal, perfectly normal, first countable perfectly normal, paracompact, first countable paracompact, or metric. In this note we show that for $P=$ Urysohn or first countable Urysohn, a $P$-closed space may not be Katětov- $P$; this also answers a question posed in [4].

Construction of the SPACE $(X, \tau)$. Let $I_{1}, I_{2}, I_{3}$, and $I_{4}$ be pairwise disjoint, dense subsets of the unit interval $I$ whose union is $I$, $I_{5}=I_{1}$, and $X=\mathrm{U}_{i=1}^{5} I_{i} x\{i\}$. A set $U$ is open precisely when

(a) for $i=1,3$, or $5,(x, i) \in U$ if and only if there is an open set $V$ in $I$ (usual topology) such that $(x, i) \in V x\{i\} \cap X \subseteq U$, and

(b) for $i=2$ or $4,(x, i) \in U$ if and only if there is an open set $V$ in $I$ such that $(x, i) \in V x\{i-1, i, i+1\} \cap X \subseteq U$.

Clearly, $(X, \tau)$ is first countable, semiregular, and Urysohn.

For $\epsilon>0$ and $x \in I$, let $S_{\epsilon}(x)$ denote the open interval $(x-\epsilon, x+\epsilon)$.

Lemma. Let $\sigma$ be a Urysohn topology on the set $X$ such that $\sigma \subseteq \tau$. (a) If $a \leqq b$ are real numbers, then the subspaces

Received by the editors September 15, 1969.

AMS Subject Classifications. Primary 5423, 5452.

Key Words and Phrases. Minimal topological spaces, minimal Urysohn spaces, Urysohn-closed spaces, Katětov-Urysohn spaces, Urysohn spaces, separation axioms.

1 This research was partially supported by a University of Kansas Research Grant No. 3416-5038. 


$$
\bigcup_{i=1}^{4}\left([a, b] \cap I_{i}\right) x\{i\} \text { and } \bigcup_{i=2}^{5}\left([a, b] \cap I_{i}\right) x\{i\}
$$

are Urysohn-closed.

(b) For $x \in I$ and $\epsilon>0, \bigcup_{i=1}^{5}\left(S_{\epsilon}(x) \cap I_{i}\right) x\{i\} \in \sigma$.

(c) For $i=1$ or 5 ,

$$
\mathrm{Cl}_{\sigma}\left(\left(S_{\epsilon}(x) \cap I_{i}\right) x\{i\}\right) \subseteq \bigcup_{j=1}^{5}\left([x-\epsilon, x+\epsilon] \cap I_{j}\right) x\{j\} .
$$

(d) Let $x \in I_{i}$ where $i=1$ or 5 . $A$ set $A \subseteq X$ is a $\sigma$-neighborhood of $(x, i)$ if and only if there is $\epsilon>0$ such that $\left(S_{\epsilon}(x) \cap I_{i}\right) x\{i\} \subseteq A$.

Proof. If for a space $(Y, \rho), \rho_{s}$ denotes the topology on $Y$ which has $\left\{(\bar{T})^{0} \mid T \in \rho\right\}$ as a base, then one can readily verify that $(Y, \rho)$ is Urysohn-closed if and only if $\left(Y, \rho_{s}\right)$ is Urysohn-closed. If $(Y, \rho)$ is a space in (a), then one can show that $\left(Y, \rho_{s}\right)$ is homeomorphic with a Urysohn-closed space that is produced by a construction in [5, Example 5]. (b) follows from (a), (c) follows from (b), and (d) follows from (a) and (b).

Theorem. For $P=$ Urysohn or first countable Urysohn, $(X, \tau)$ is $P$-closed but not Katětov-P.

Proof. By (a) of the lemma, it follows that $(X, \tau)$ is Urysohnclosed. Assume that $(X, \tau)$ is Katětov-Urysohn. Then there is a coarser minimal Urysohn topology $\sigma$ on $X$. Let $x \in I_{2}$ and fix $i \in\{1,5\}$. Using (c) of the lemma, it is easy to verify that the topology $\sigma_{x}^{i}$ defined on $X$ by $U \in \sigma_{x}^{i}$ precisely when $U \in \sigma$ and $(x, 2) \in U$ implies there is an $\epsilon>0$ such that $\left(S_{\epsilon}(x) \cap I_{i}\right) x\{i\} \subseteq U$ is a coarser Urysohn topology; hence, $\sigma=\sigma_{x}^{i}$ for each $x$ in $I_{2}$. Let $y \in I_{1}$. There is $\epsilon>0$ such that for $U_{i}=\left(S_{\epsilon}(y) \cap I_{i}\right) x\{i\}$ for $i=1$ and $5, \mathrm{Cl}_{\sigma}\left(U_{1}\right) \cap \mathrm{Cl}_{\sigma}\left(U_{5}\right)=\varnothing$. Since $I_{2}$ is dense in $I$, there is a point $z$ in $S_{\epsilon}(y) \cap I_{2}$. Since $\sigma_{z}^{i}=\sigma$ for $i=1$ and 5 , then $(z, 2) \in \mathrm{Cl}_{\sigma}\left(\left(S_{\epsilon}(y) \cap I_{i}\right) x\{i\}\right)$ for $i=1$ and 5 , a contradiction. This proves the theorem for the case $P=$ Urysohn. The case $P=$ first countable Urysohn follows from the fact that $(X, \tau)$ is first countable and the proof of the case $P=$ Urysohn.

A space is completely Hausdorff if and only if for every pair, $x, y$ of distinct points, there exists a real-valued continuous function $f$ such that $f(x) \neq f(y)$. As far as the author knows, the question for $P=$ regular and $P=$ first countable completely Hausdorff is unsolved. 


\section{REFERENCES}

1. B. Banaschewski, Über zwei Extremaleigenschaften topologischer Räume, Math. Nachr. 13 (1955), 141-150. MR 17, 66.

2. - On the Weierstrass-Stone approximation theorem, Fund. Math. 44 (1957), 249-252. MR 19, 1182.

3. M. P. Berri, Minimal topological spaces, Trans. Amer. Math. Soc. 108 (1963), 97-105. MR 27 \#711.

4. M. P. Berri, J. R. Porter and R. M. Stephenson, Jr., A survey of minimal topological spaces, Proc. Kanpur Topological Conference, 1968 (to appear).

5. H. Herrlich, $T_{\nu}$-Abgeschlossenheit und T $T_{\nu}$-Minimalität, Math. Z. 88 (1965), 285-294. MR 32 \#1664.

6. M. Katětov, Über H-abgeschlossene und bikompakte Räume, Casopis Pěst. Mat. Fys. 69 (1940), 36-49. MR 1, 317.

7. C. T. Scarborough, Minimal Urysohn spaces, Pacific J. Math. 27 (1968), 611617. MR $38 \# 6530$.

8. C. T. Scarborough and R. M. Stephenson, Jr., Minimal topologies, Colloq. Math. 19 (1968), 215-219. MR 37 \#3522.

9. N. Smythe and C. A. Wilkins, Minimal Hausdorff and maximal compact spaces, J. Austral. Math. Soc. 3 (1963), 167-171. MR 27 \#4204.

10. R. M. Stephenson, Jr., Minimal first countable topologies, Trans. Amer. Math. Soc. 138 (1969), 115-127. MR 38 \#6537.

University of Kansas, Lawrence, Kansas 66044 